$29.6 \%, p=0.026$ ) (Table 1.). No statistically significant differences were found in the percentages or the absolute numbers of T, B or NK cells.

Conclusions: Our data support previous reports indicating that depletion of lymphocyte in the PB of SSc patients. However, we found no significant difference in relation to lymphocyte subtypes, which differs from the literature data. References:

[1] T and NK Cell Phenotypic Abnormalities in Systemic Sclerosis: a Cohort Study and a Comprehensive Literature Review. Almeida et al, 2015.

[2] Liu M, Wu W, Sun X, Yang J, Xu J, Fu W, Li M. New insights into CD4+ T cell abnormalities in systemic sclerosis. Cytokine \& Growth Factor Reviews, 2016; 28:31-36.

[3] Gambichler T, Tigges C, Burkert B, Höxtermann S, Altmeyer P, Kreuter A. Absolute count of $T$ and $B$ lymphocyte subsets is decreased in systemic sclerosis. Eur J Med Res 2010; 15:44-46.

Disclosure of Interest: None declared

DOI: 10.1136/annrheumdis-2017-eular.7028

\section{AB0175 INHIBITORY EFFECT OF ENDOTHELIN-1 TYPE A RECEPTOR ANTAGONISTS ON MIGRATION OF NEUTROPHILS AND TUMOUR CELLS}

L.M. Kappes ${ }^{1}$, S. Sommerlatte ${ }^{1}$, C. Plattfaut ${ }^{2}$, S. Pitann ${ }^{1}$, G. Marschner ${ }^{1}$, H. Heidecke ${ }^{3}$, P. Lamprecht ${ }^{1}$, A. Müller ${ }^{1}$, F. Gieseler ${ }^{2}$, O. Cabral-Marques ${ }^{1}$, G. Riemekasten ${ }^{1}$. ${ }^{1}$ Department of Rheumatology; ${ }^{2}$ Department of Oncology, University of Lübeck, Lübeck; ${ }^{3}$ Celltrend GmbH, Luckenwalde, Germany

Background: Endothelin-1 type A receptor (ETAR) antagonists (e.g. ambrisentan) are currently approved by the U.S Food and Drug administration, representing a well-tolerated treatment of pulmonary arterial hypertension (PAH)[1] for patients with connective tissue diseases such as systemic sclerosis (SSc). Noteworthy, increased numbers of infiltrating neutrophils have been associated with worse clinical outcome in PAH patients. In another context, several studies have reported that endothelin-1 and its receptor ETAR also play a central role in the development of tumour cell invasion and metastasis. However, the effects of ETAR antagonists on migration of neutrophils and tumour cells remain to be determined.

Objectives: The objective was to analyse the effects of two ETAR antagonists on migration of neutrophils and tumour cell lines.

Methods: The migratory ability of peripheral neutrophils from healthy donors (HD) and different tumour cell lines (myeloid leukaemia HL60 cells and human pancreatic adenocarcinoma COLO357 cells) was analysed in response to $\mathrm{N}$ Formylmethionyl-leucyl-phenylalanine (FMLP) or Protease-activated receptor 2 (Par-2) agonist. Because it has been shown before [2], IgGs from HD and SSc patients were used as additional stimulus for migration. Neutrophils and HL60 cells were preincubated (1h) with sitaxentan or ambrisentan, respectively, before being tested for migration (1h) using the Transwell assay. COLO357 cells were incubated (48h) in the presence of sitaxentan and migration was tested in the Oris Pro Cell assay. Migration was analysed by automatic cell counting or digital photo analysis and a migration index was calculated.

Results: Sitaxentan and/or ambrisentan significantly blocked the migration of neutrophils and tumour cell lines. In more detail, neutrophil migration in response to FMLP, being set to $100 \%$, was completely inhibited by sitaxentan $(0,46 \%)$. Further, neutrophil migration in response to IgGs from HD and SSc patients was induced equally, again being set to $100 \%$. In the presence of sitaxentan migration was reduced to $60 \%$, respectively. In DMSO-differentiated HL60 cells the migratory capacity in response to FMLP $(100 \%)$ was reduced to $66 \%$ by ambrisentan and to $14 \%$ by sitaxentan. Moreover, in the presence of sitaxentan and a Par-2 agonist the migratory ability of COLO357 cells was significantly decreased to $89 \%$ compared to Par-2 agonist only, being set to $100 \%$.

Conclusions: Our results suggest a pivotal and non-redundant role of ETAR in cell migration, which needs further clarification in order to repurpose the use of ETAR inhibitors. Therapeutic switching of ETAR antagonists from PAH to cancer therapies is a promising adjuvant therapy.

\section{References:}

[1] Waxman AB. A review of sitaxsentan sodium in patients with pulmonary arterial hypertension. Vasc Health Risk Manag 2007;3:151-7.

[2] Kill A, Tabelin C, Undeutsch R, et al. Autoantibodies to angiotensin and endothelin receptors in systemic sclerosis induce cellular and systemic events associated with disease pathogenesis. Arthritis R\&T, 2014, 16:R29.

Disclosure of Interest: None declared

DOI: 10.1136/annrheumdis-2017-eular.6173

\section{AB0176 MITOCHONDRIAL DYSFUNCTION IN IDIOPATHIC INFLAMMATORY MYOPATHY DERIVED MYOBLASTS}

C. Basualto-Alarcón ${ }^{1}$, M.F. Bozán ${ }^{2}$, J. Bevilacqua ${ }^{1,2}$, F. Urra ${ }^{1}$, D. González ${ }^{1}$, H. Gatica ${ }^{2}$, A. Göecke ${ }^{2}$, P. Wurmann ${ }^{2}$, M.F. Sabugo ${ }^{2}$, S. Saavedra ${ }^{2}$, C. Cárdenas ${ }^{1,3,4} .{ }^{1}$ Cellular Metabolism and Bioenergetics Laboratory; Anatomy and Developmental Biology Program; ICBM; Faculty of Medicine., Universidad de Chile; ${ }^{2}$ Hospital Clínico Universidad de Chile; ${ }^{3}$ Geroscience Center for Brain Health and Metabolism, Universidad de Chile, Santiago, Chile; ${ }^{4}$ Buck Institute for Research on Aging, Novato, United States

Background: Idiopathic inflammatory myopathies (IIM) are acquired skeletal muscle diseases, characterized by proximal muscle weakness. This syndrome includes five different (1) diseases, nevertheless, in this work, we included dermatomyositis (DM) and polymyositis (PM) patients. Has been reported that despite treating inflammation, muscle atrophy and weakness persist in some patients, suggesting an inherent muscle cause $(2,3)$. In addition, histological studies show some mitochondrial abnormalities. Our aim was to evaluate a possible role for mitochondrial dysfunction in the pathophysiology of this diseases. Objectives: To compare the mitochondrial status of myoblast obtained from myositis patients -and healthy control- biopsies.

Methods: Primary cultured myoblasts extracted from the deltoid of IIM patients were used and compared with myoblasts obtained from normal patients subjected to shoulder surgery. Also, a human skeletal muscle cell line (RCMH) was used as control. The bioenergetic profile was analyzed with an Extracellular flux analyzere 96 (Seahorse Biosciences). Also, biopsy tissue was used for Western blot (WB) and immunofluorescence experiments.

Results: Basal oxygen consumption rate, ATP-linked oxygen consumption, maximal oxygen consumption and spare respiratory capacity were lower in IIM myoblasts when compared to RCMH myoblasts, however, when compare to control primary cultured myoblasts we only find differences in ATP-linked oxygen consumption. Expression levels of mitochondrial complexes (I, III, IV and V) were analyzed by WB in tissue samples. No differences were observed between control and IIM patients. Mitochondrial area was estimated by immunofluorescence of the voltage dependent anion channel (VDAC), which show no differences between control and patients.

Conclusions: IIM derived myoblasts present a compromised mitochondrial function, compared to control myoblasts. Specially, oxygen consumption associated with ATP synthesis show decreased levels in patients. Although, expression levels of the mitochondrial complexes as well as mitochondrial area were not different between control and patients. Future experiments should address if IIM mitochondria are consuming less oxygen because of a lower ATP demand or because a primary mitochondrial damage.

\section{References:}

[1] Dalakas M. Inflammatory Muscle Diseases. N Engl J Med. 372:1734-1747, 2015.

[2] Park, J. H. et al. Magnetic resonance imaging and P-31 magnetic resonance spectroscopy provideunique quantitative data useful in the longitudinal management of patients with dermatomyositis. Arthritis Rheum.37, 736-46, 1994.

[3] Lee, H.-K. et al. Foxo/Atrogin induction in human and experimental myositis. Neurobiol. Dis.46, 463-475, 2012.

Acknowledgements: FONDECYT 3150623 \& 1151383; FONDAP 15150012.

Disclosure of Interest: None declared

DOI: 10.1136/annrheumdis-2017-eular.3889

\section{AB0177 INCREASED LEVELS OF THE INFLAMMATORY PROTEINS CXCL10, CXCL11, TNFR2 AND YLK-40 TYPIFY THE EARLIEST PHASE OF SYSTEMIC SCLEROSIS (SSC)}

M. Cossu $^{1,2}$, L. van Bon ${ }^{1}$, M. Rossato ${ }^{1,2}$, L. Beretta ${ }^{3}$, T.R.D.J. Radstake ${ }^{1,2}$ ${ }^{1}$ Rheumatology \& Clinical Immunology; ${ }^{2}$ Laboratory of Translational Immunology (LTI), UMC Utrecht, Utrecht, Netherlands; ${ }^{3}$ Referral Center for Systemic Autoimmune Diseases, Fondazione IRCCS Ca' Granda Ospedale Maggiore Policlinico, Milano, Italy

Background: Definite systemic sclerosis (defSSc) patients yet lacking the prototypical signs of fibrosis stratify in an intermediate severity stage between pre-clinical and fibrotic, limited cutaneous (IcSSc) and diffuse cutaneous (dcSSc) phenotypical subsets ${ }^{1}$.

Objectives: We aimed to molecularly position defSSc patients in respect to healthy controls (HC) and early SSc (EaSSc) patients with a broad panel of serum mediators of inflammation and tissue damage in an attempt to increase the knowledge in pathophysiologic mechanisms and biomarkers availability, thereby offering new ground for disease interception before fibrosis develops.

Methods: To this end, an 88-plex immunoassay was performed in sera from an identification cohort composed of 21 EaSSc according to LeRoy and Medsger criteria $^{2}$ without other signs and symptoms of evolutive disease, 15 defSSc patients according to the 2013 ACR/EULAR criteria ${ }^{3}$ without skin or lung fibrosis and $11 \mathrm{HC}$. A larger cohort comprising $47 \mathrm{EaSSc}, 48$ defSSc and $43 \mathrm{HC}$ was used for replication purposes. Fifty-one IcSSc and 35 dcSSc as comparison with established, fibrotic disease were recruited in parallel.

Results: Sixteen mediators differentially expressed in EaSSc and defSSc were selected for replication (one-way ANOVA and/or ANOVA polynomial test for trend with exploratory threshold $\mathrm{p}<0.1)$. Amongst these, after correction for multiple comparisons, CXCL10/IP-10, CXCL11/I-TAC, TNFR2 and CHI3L1/YKL40 showed a significant upregulation in defSSc and EaSSc with a linear increase from $\mathrm{HC}$ to EaSSc to defSSc. The level of upregulation observed in defSSc individuals was similar (CXCL10/IP-10, CXCL11//IAC) or further increased (TNFR2, CHI3L1/YKL-40) in IcSSc and dcSSc patients. A set of 7 ranked markers (Angiopoietin-2, TNFR2, CXCL11/I-TAC, CXCL10/IP-10, sICAM-1, CHI3L1/YKL40, CXCL9/MIG) provided a good visualization of the gradually increasing pattern from $\mathrm{HC}$ to EaSSc to defSSc to IcSSc and dcSSc.

Conclusions: This is the first attempt to validate circulating biomarkers defining the earliest phases of SSc. Despite the need for confirmation in a prospective 
setting, our results confirm that defSSc might represent an intermediate entity between pre-clinical stages and the most severe subsets of disease, thereby opening new perspectives on SSc pathophysiology and disease interception.

[1] Cossu M, et al. Rheumatology 2016

[2] LeRoy EC, et al. J Rheumatol 2001.

[3] van den Hoogen F, et al. Arthritis Rheum 2013.

Acknowledgements: Supported by a grant from Gruppo Italiano per la Lotta alla Sclerodermia (GILS). MC and TR are partly supported by the VIDI laureate and Dutch Arthritis Foundation (NWO, Netherlands Institute for Science) and ERC starting grant (EU) obtained by TR. The LTI MultiPlex Core Facility is acknowledged for technical performance of the multiplex immune assays.

Disclosure of Interest: None declared

DOI: 10.1136/annrheumdis-2017-eular.5295

\section{AB0178 PHENOTYPING OF NATURAL KILLER (NK) RECEPTORS ON NK AND NKT-LIKE CELLS DISCLOSES DEFECTIVE IMMUNE-REGULATORY CAPABILITY IN PATIENTS WITH SYSTEMIC SCLEROSIS}

M. Cossu $^{1,2}$, L. Beretta ${ }^{3}$, E. Trombetta ${ }^{4}$, S.T.A. van Bijnen ${ }^{5,6}$, M. Rossato ${ }^{1,2}$, L. van Bon ${ }^{1}$, M. van der Kroef ${ }^{1,2}$, E.C. Chouri ${ }^{1,2}$, C.G.K. Wichers ${ }^{1,2}$,

N. Vazirpanah ${ }^{1,2}$, M.C. Vonk ${ }^{7}$, L. Porretti ${ }^{4}$, F. Preijers ${ }^{6}$, H. Dolstra ${ }^{6}$,

T.R.D.J. Radstake ${ }^{1,2} .{ }^{1}$ Rheumatology \& Clinical Immunology; ${ }^{2}$ Laboratory of Translational Immunology (LTI), UMC Utrecht, Utrecht, Netherlands; ${ }^{3}$ Referral Center for Systemic Autoimmune Diseases; ${ }^{4}$ Flow Cytometry Service, Clinical Chemistry and Microbiology Laboratory, Fondazione IRCCS Ca' Granda Ospedale Maggiore Policlinico, Milano, Italy; ${ }^{5}$ Hematology; ${ }^{6}$ Laboratory Medicine, Laboratory of Hematology; ${ }^{7}$ Rheumatology, Radboud University Medical Center, Nijmegen, Netherlands

Background: Systemic sclerosis (SSc) is an autoimmune disease characterized by dysregulation of the immune system, vasculopathy and fibrosis of the skin and internal organs. Natural Killer $\left(C D 56^{+}\right.$CD3-, NK) and NKT-like $\left(C D 56^{+} \mathrm{CD}^{+}\right)$cells display receptors (NKR) whose expression pattern determines their cytotoxic and immune-regulatory activity. The role of NK and NKT-like cells in the dysregulation of the immune system in SSc has not been fully elucidated yet.

Objectives: To improve our knowledge on the contribution of NK, NKT-like and a subset of NKT cells expressing invariant TCR (iNKT) in SSc development, we performed a broad phenotyping of NKR in the circulation of SSc patients, including subjects with pre-clinical SSc.

Methods: NKR were assessed by flow cytometry using two 13-color panels on whole blood of 84 SSc patients and 20 healthy controls (HC). In particular, 15 patients with early SSc (EaSSc) without signs or symptoms of evolutive disease (2001 LeRoy and Medsger criteria ${ }^{1}$ ), 24 patients with definite SSc without skin or lung fibrosis (defSSc), 26 patients with limited (IcSSc) and 19 patients with diffuse cutaneous SSc (dcSSc) (2013 ACR/EULAR classification criteria for SSc $^{2}$ ) were included. NK degranulation in response to K562 target cells was assessed in IcSSc and dcSSc patients versus HC

Results: The number of circulating lymphocytes, NKT-like and iNKT cells - but neither $\mathrm{CD}^{+} \mathrm{T}$ nor NK cells - was reduced in dcSSc versus HC. NKp46+ NK cells co-expressing NKG2D and CD16 were decreased in dcSSc versus $\mathrm{HC}$ and EaSSc. Consistently with these observations, dcSSc exhibited lower degranulation capability. $\left(\mathrm{CD} 57^{+}\right) \mathrm{KIR}^{+}$and activating NKR-expressing NKT-like cells were diminished in both dcSSc and IcSSc versus $\mathrm{HC}$.

Conclusions: dcSSc patients showed a defective NK cytotoxicity potential, possibly due to the decreased $\mathrm{NKp}_{4} 6^{+}$fraction. The regulatory, cytolytic $\mathrm{KIR}^{+}$ NKT-like fraction was also reduced with a parallel decrease of activating receptors expression in both IcSSc and dcSSc. Overall these results point towards an impairment of NK and NKT-like cells as immune check-points in fibrotic SSc.

References:

[1] LeRoy EC, et al. J Rheumatol 2001.

[2] van den Hoogen F, et al. Arthritis Rheum 2013

Acknowledgements: Supported by a grant from Gruppo Italiano per la Lotta alla Sclerodermia (GILS). MC and TR are partly supported by the VIDI laureate and Dutch Arthritis Foundation (NWO, Netherlands Institute for Science) and ERC starting grant (EU) obtained by TR. The authors would like to thank Koos Gaiser (U-DAIR, LTI, UMC Utrecht) for the technical assistance in developing the 13-color flow cytometry panels; Dr F. Montero, L. Nieto-Gligorovski and E. Gautherot (Beckman Coulter Inc, Marseille) for providing the NKG2A-PB antibody; the NIH Tetramer Facility for providing the CD1d tetramers.

Disclosure of Interest: None declared

DOI: 10.1136/annrheumdis-2017-eular.5059

\section{AB0179 DEGRADATION OF TYPE VII COLLAGEN (C7M) IS ASSOCIATED WITH SYSTEMIC SCLEROSIS - DEVELOPMENT OF A NOVEL NEO-EPITOPE SPECIFIC ASSAY}

J. Sand ${ }^{1}$, P. Juhl ${ }^{1}$, L. Iversen ${ }^{2}$, T. Karlsmark ${ }^{2}$, M. Karsdal ${ }^{1}$, A.-C. Bay-Jensen ${ }^{1}$, M. Mogensen ${ }^{2}$, A.S. Siebuhr ${ }^{1}$, D. Leeming ${ }^{1}$. ${ }^{1}$ Nordic Bioscience, Herlev;

${ }^{2}$ Department of dermatology, Bispebjerg Hospital, Copenhagen, Denmark

Background: Type VII collagen (col7) is the main component of the anchoring fibrils that connects the basement membrane to the underlying interstitial matrix and has mainly been investigated for its role in blistering skin diseases. It has been investigated for its role in dystrophic epidermolysis bullosa, a severe skin disease. Furthermore, increased levels of type VII collagen in skin has been reported for patients with systemic sclerosis (SSc).

Objectives: The objective was to develop and characterize a blood-based marker assessing col7 degradation in patients with SSc.

Methods: We identified a specific fragment of col7 in serum from COPD patients, which was not found in controls, using mass spectrometry. A monoclonal antibody was raised against the first ten amino acids of the neo-epitope (KLH-CGGGPPGPPGRLV) and employed in a competitive ELISA (C7M). The C7M assay was validated technically and was subsequently evaluated in 2 cohorts including SSc patients. The first cohort (SSc\#1; $n=35)$ consisted of early $(<2$ years of SSc symptoms; $n=16)$ and late ( $>10$ years of disease with stable skin for at least 6 months, $n=19$ ) diffuse SSc patients, while the second cohort (SSc\#2; $n=119)$ consisted of limited $(n=78)$ and diffuse $(n=41)$ SSc patients. Serum C7M levels were likewise measured in healthy subjects and compared to the levels of SSc patients using the Kruskal-Wallis test with Dunn's multiple comparisons test comparing healthy individuals with the two SSc cohorts.

Results: A technically robust competitive ELISA (C7M), which was highly specific for a col7 fragment was developed. The assay showed acceptable inter- $(13 \%)$ and intra-assay ( $9 \%)$ variation, linearity (102\% dilution recovery), analyte stability (102\% recovery after 4 freeze/thaw cycles), and interference.

The C7M marker was evaluated by comparing serum levels in healthy donors with patients with SSc (Figure). Serum C7M levels were not associated with age, gender, BMI, or disease duration. The geometric mean serum C7M level in healthy donors was $4.6 \mathrm{ng} / \mathrm{mL}(95 \% \mathrm{Cl} 3.7-5.6 \mathrm{ng} / \mathrm{mL})$. The geometric mean serum C7M levels were significantly elevated in both cohorts of patients with SSc (SSc\#1, $13.6 \mathrm{ng} / \mathrm{mL}[95 \% \mathrm{Cl} 11.1-16.5], \mathrm{p}<0.0001 ;$ SSc\#2, $9.2 \mathrm{ng} / \mathrm{mL}[95 \%$ $\mathrm{Cl}$ 8.3-10.2], $\mathrm{p}<0.0001)$. Furthermore, a significant difference were observed between the two cohorts $(P=0.05)$.

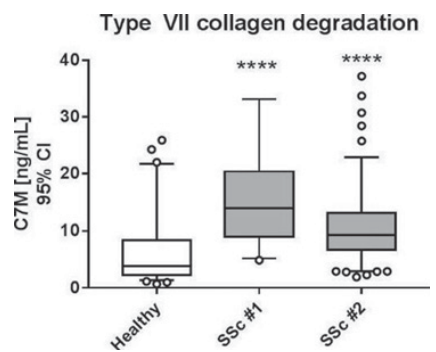

Conclusions: The C7M ELISA enabled quantification of type VII collagen degradation in serum. Elevated serum C7M levels indicated that the remodeling of type VII collagen was significantly increased in patients with SSc, suggesting a pathological role.

Acknowledgements: We thank Biogen Idec's SSc department for their contribution.

Disclosure of Interest: None declared

DOI: 10.1136/annrheumdis-2017-eular.5689

\section{AB0180 BIOMARKERS OF EXTRACELLULAR MATRIX REMODELING ARE ASSOCIATED WITH ACUTE EXACERBATIONS OF IDIOPATHIC INTERSTITIAL PNEUMONIA}

J. Sand ${ }^{1}$, P. Juhl ${ }^{1}$, Y. Tanino ${ }^{2}$, T. Nikaido ${ }^{2}$, K. Misa ${ }^{2}$, Y. Sato ${ }^{2}$, R. Togawa ${ }^{2}$ X. Wang ${ }^{2}$, M. Karsdal ${ }^{1}$, A.-C. Bay-Jensen ${ }^{1}$, D. Leeming ${ }^{1}$, A.S. Siebuhr ${ }^{1}$, M. Munakata ${ }^{2} .{ }^{1}$ Nordic Bioscience, Herlev, Denmark; ${ }^{2}$ Department of Pulmonary Medicine, Fukushima Medical University School of Medicine, Fukushima, Japan

Background: Idiopathic interstitial pneumonia (IIP) is characterized by an increased rate of extracellular matrix (ECM) turnover resulting in fibrosis. The pathogenic remodeling includes increased levels of protein synthesis and degradation mediated by proteases such as matrix metalloproteinases (MMPs). Acute exacerbations of IIP (AE-IIP) represent periods of increased disease activity. Pulmonary involvement, especially pulmonary fibrosis, is common in patients suffering from systemic sclerosis (SSc) and ankylosing spondylitis (AS)

Objectives: The objective was to investigate if ECM remodeling was altered during AE-IIP by serological neo-epitope biomarkers.

Methods: Serum samples were collected from patients with IIP at clinically stable disease (S-IIP, $n=29)$ and at AE-IIP $(n=68)$. Of these, 11 and 28 patients, respectively, had idiopathic pulmonary fibrosis (IPF). 28 IIP patients had paired samples. Biomarkers released from MMP-mediated degradation of collagen type I (C1M), III (C3M), IV (C4M), and VI (C6M), elastin (ELM7), versican (VCANM), biglycan (BGM), and C-reactive protein (CRPM) were assessed in serum by competitive ELISAs utilizing neo-epitope specific monoclonal antibodies. Data were analysed using Mann-Whitney test, Wilcoxon test, Spearman's rank correlation, and Kaplan-Meier curves as appropriate.

Results: Mean age of patients was 71 (range 54-86) at AE-IIP and 69 (range 55-83) at S-IIP. Mean forced vital capacity in percentage of predicted value (\%FVC) was $55.6 \%($ SD 19.5) at AE-IIP and $79.0 \%$ (SD 26.5) at S-IIP. Serum 